\title{
Development of a new tetrafunctional hybrid to target cancer cells
}

\author{
Maya Georgieva ${ }^{1}$, Androniki Kostagianni ${ }^{2}$, Eirinaios Vrettos ${ }^{2}$, Maria Chatziathanasiadou', Andreas Tzakos $^{2}$, \\ Tamara Pajpanova ${ }^{1}$
}

\author{
${ }^{1}$ Institute of Molecular Biology Roumen Tsanev, Bulgarian Academy of Sciences, Bulgaria \\ ${ }^{2}$ University of Ioannina, Department of Chemistry, Section of Organic Chemistry and Biochemistry, Greece
}

https://doi.org/10.17952/35EPS.2018.325

\section{Introduction}

Peptide sequences AVPI- and RGD- are interesting subject of targeted drug design in recent years. AVPI (Ala-Val-Pro-Ile) motif is the functionally essential sequence of the $\mathrm{N}$-terminus of pro-apoptotic regulator Smac (Second mitochondria derived activator of caspases). It is shown that different AVPI- and Smacmimetics could help proceeding of extrinsic and intrinsic apoptotic pathways by inhibition of IAP proteins (Inhibitor of Apoptosis Proteins) [1,2]. An inverse correlation between Smac and IAP protein levels in a wide range of cancer cells has been reported [2]. RGD (Arg-Gly-Asp) is known for its targeting potential to cancerous cells by binding to molecules over-expressed on their cell membranes [3].

Combining of several motifs with different functional activities into single molecule is an advantageous strategy in drug development in recent decades. Herein, we designed a novel tetra-functional peptide hybrid containing Smac peptide fragment along with RGD targeting unit, a unit for conjugation of drugs/dyes and canavanine-tail in order to lower down the apoptotic thresholds and trigger apoptosis in cancer cells.

\section{Results and Discussion}

In order to enhance the anti-proliferative potential of AVPI motif and its cell membrane permeability we designed the following peptide:

We modified the $\mathrm{A}^{1} \mathrm{~V}^{2} \mathrm{P}^{3} \mathrm{I}^{4}$ sequence by incorporating L-hydroxyproline instead of proline at the 3rd position. Then lysine residue was attached as a site for further conjugations regarding the data that free $\mathrm{N}$-terminal $\mathrm{NH}_{2}$ group is needed for more effective functional binding of AVPI sequence. RGD delivering motif was also attached. Then a tail of three canavanines was incorporated at the C-terminus of the peptide (Fig. 1). Cell permeability predictions pointed us that at least three arginine residues in a row are needed for good CPP index of the designed peptide. Instead of arginine we used L-canavanine, the $\delta$-oxa analogue of L-arginine. Canavanine is a non-proteinogenic arginine analogue that shows cell viability inhibitory effect on transformed cells [4.5].

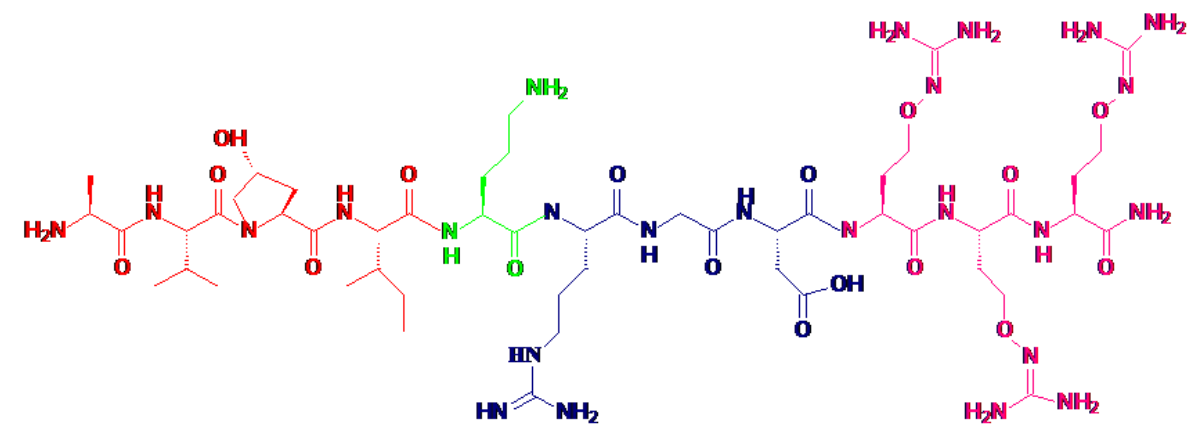

Figure 1: Tetra-functional peptide

The peptide we synthesized by manual standard Fmoc solid-phase protocol. Commercially available Rink-Amide resin $(0.59 \mathrm{mmol} / \mathrm{g}$ substitution)was used. The coupling of each amino acid was performed in the presence of 3 mol excess of: Fmoc-amino acid, HOBt, DIC, in a solution of DMF:DCM (1:1). The successful coupling and the following Fmoc-deprotection were monitored by Kaiser test. Fmoc-groups were removed with solution of 20\% Piperidine in DMF with HOBt (0.1M final concentration). Cleavage step from the resin and side chain protecting groups was accomplished by treatment with $10 \mathrm{ml}$ of TFA/dH2O/TIS cocktail ( $95 \%: 2.5 \%: 2.5 \%$ ). 
Crude peptide was purified by semi-preparative HPLC (Fig.2) and characterized by LC/MS.

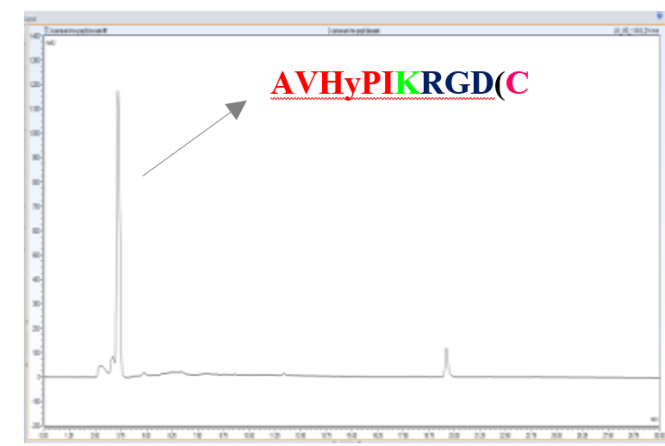

Figure 2: HPLC chromatogram of the crude peptide; Conditions: Gradient: $10 \%-100 \%$ ACN + 0.1\% TFA; Time: 30 min; Detection: $214 \mathrm{~nm}$

Effect of the peptide on A549 cells

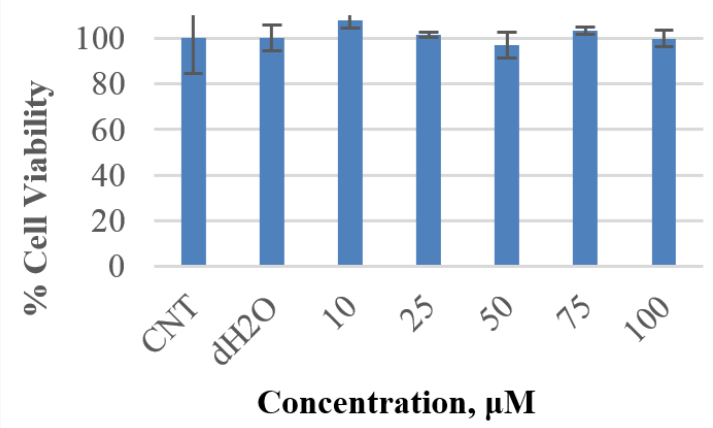

Figure 3: Effect of the peptide on A549 cells after $72 \mathrm{~h}$ treatment. Each concentration was performed in triplicates.

The anti-proliferative activity of the pure peptide was tested over A549 lung cancer cell line by MTT assay. We found that that the peptide had no effect in the tested concentrations, when it was used as single agents (Fig. 3). Still that is in concert with the literature data describing AVPI-mimetics also as sensitizing agents to different used in practice anticancer drugs [6]. In summary, for first time it was synthesized an AVPI-mimetic with three-canavanine tail used as penetrating vector. The penetration potential of the peptide is under examination.

\section{Acknowledgments}

The study was supported by National Science Fund, Ministry of Education and Science, Bulgaria; Project: DN19/17, 20.12.2017; EPS Mobility Fellowship 2018.

\section{References}

1. Chen, D., and Huerta S. J. Anti-Cancer Drugs 20, 646-658 (2009).

2.. Martinez-Ruiz, G., et al. J Experimental and Clinical Cancer Research 27:48 (2008), .

3. Boohaker, R.J. et al., Curr Med Chem 19 (22), 3794-3804 (2012).

4. Bence, A. and Crooks P, J Enzyme Inhibition and Medical Chemistry 18, 383-394 (2003).

5. Park, H., et al. J. Life Science 19, 1529-1537 (2009).

6. Greer, R., et al. Cancer Res. 71 (24), 7640-7648 (2011). 